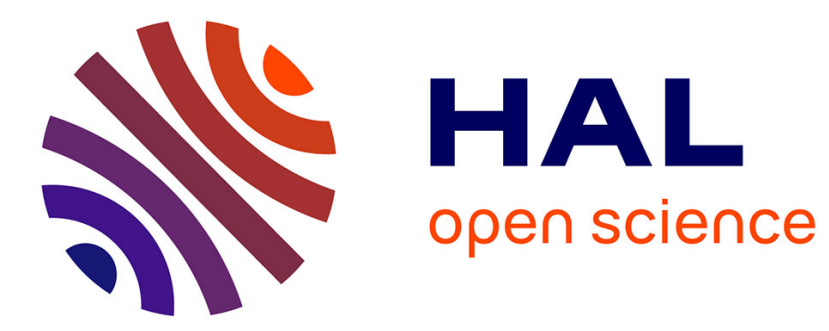

\title{
Analyticity angle for non-commutative diffusion semigroups
}

Christoph Kriegler

\section{To cite this version:}

Christoph Kriegler. Analyticity angle for non-commutative diffusion semigroups. Journal of the London Mathematical Society, 2010. hal-03123937

\section{HAL Id: hal-03123937 \\ https://hal.science/hal-03123937}

Submitted on 28 Jan 2021

HAL is a multi-disciplinary open access archive for the deposit and dissemination of scientific research documents, whether they are published or not. The documents may come from teaching and research institutions in France or abroad, or from public or private research centers.
L'archive ouverte pluridisciplinaire HAL, est destinée au dépôt et à la diffusion de documents scientifiques de niveau recherche, publiés ou non, émanant des établissements d'enseignement et de recherche français ou étrangers, des laboratoires publics ou privés. 


\title{
ANALYTICITY ANGLE FOR NON-COMMUTATIVE DIFFUSION SEMIGROUPS
}

\author{
CHRISTOPH KRIEGLER
}

\begin{abstract}
Under certain hypotheses, diffusion semigroups on commutative $L^{p}$-spaces are known to have an analytic extension for $|\arg z|<\frac{\pi}{2}-\arctan \frac{|p-2|}{2 \sqrt{p-1}}$. In this paper it is shown that semigroups on non-commutative $L^{p}$-spaces have the same extension under suitable conditions. These conditions even lead to a new result in the commutative case. Further, some examples are considered.
\end{abstract}

2000 Mathematics Subject Classification : 47D07, 46L52.

\section{INTRODUCTION}

The spectral theory of (generators of) diffusion semigroups $\left(T_{t}\right)$ on commutative (i.e. classical) $L^{p}$-spaces has been studied in a series of articles $[1,23,22,31,17,18]$. Here we follow Stein's classical work [28] and mean by the term diffusion the fact that $T_{t}$ is contractive as an operator $L^{p} \rightarrow L^{p}$ for all $p \in[1, \infty]$ and self-adjoint on $L^{2}$ (see 4.1 for the exact definition). Such a semigroup has an analytic extension on $L^{2}$ to the right half plane. Then it follows from a version of Stein's complex interpolation [32] that there is an analytic extension on $L^{p}$ to a sector in the complex plane, symmetric to the real axis and with half opening angle

$$
\frac{\pi}{2}-\pi\left|\frac{1}{p}-\frac{1}{2}\right|
$$

In [22], it is shown with a different method that this angle can be enlarged.

Theorem 1.1. [22, cor 3.2] Let $\left(T_{t}\right)_{t \geq 0}$ be a diffusion semigroup on some $\sigma$-finite measure space, i.e.

(1) $\left\|T_{t}: L^{p} \rightarrow L^{p}\right\| \leq 1$ for all $t \geq 0$ and $1 \leq p \leq \infty$,

(2) $T_{t}$ is self-adjoint on $L^{2}$.

(3) $t \mapsto T_{t}$ is strongly continuous on $L^{p}$ for $p<\infty$ and $w^{*}$-continuous for $p=\infty$.

Assume further that $T_{t} f \geq 0$ for any $f \in L^{\infty}, f \geq 0$. Then $T_{t}$ has an analytic contractive extension on $L^{p}$ to the sector

$$
\left\{z \in \mathbb{C}^{*}:|\arg z|<\frac{\pi}{2}-\arctan \frac{|p-2|}{2 \sqrt{p-1}}\right\}
$$

Date: April 24, 2009.

The author is supported by the Karlsruhe House of Young Scientists and the Franco-German University DFH-UFA. 
This result is optimal. In fact, there is already a strikingly simple example on a twodimensional space with this angle (see example 4.3).

Generators of diffusion semigroups have a bounded $H^{\infty}$ functional calculus on $L^{p}$ for any $1<p<\infty$. This follows from [7]. In [19] the $H^{\infty}$ calculus angle of these operators was improved, using theorem 1.1. In the more recent past, besides vector valued spaces $L^{p}(\Omega, X)$ (see for example [11]), the attention turned to diffusion semigroups on non-commutative spaces $L^{p}(M)$ associated to a von Neumann algebra ([16], see also [14, 15]).

In this article, we consider non-commutative semigroups which are families $\left(T_{t}\right)$ of operators acting on $L^{p}(M, \tau)$ for all $1 \leq p \leq \infty$. Under reasonable hypotheses, we obtain the same sector (1.1) as in the commutative case. Our method works for hyperfinite von Neumann algebras $M$ and for semi-commutative semigroups on $L^{\infty}(\Omega) \bar{\otimes} N$, where $N$ is a QWEP von Neumann algebra.

Our assumptions are as follows. The operators $T_{t}$ are completely contractive. In the commutative case, an operator $T: L^{\infty} \rightarrow L^{\infty}$ is completely contractive iff it is contractive, so that our assumption then reduces to the classical setting. The positivity assumption in theorem 1.1 is replaced by a certain property $(\mathcal{P})$, see definition 5.1. If for example the semigroup consists of complete positive operators, this property is satisfied. In the commutative case, an operator $T: L^{\infty} \rightarrow L^{\infty}$ satisfies $(\mathcal{P})$ if and only if $T$ is contractive and extends to a self-adjoint operator on $L^{2}$ (proposition 6.1). In particular, we get theorem 1.1 without the positivity assumption, see corollary 6.2.

Note that our method (and also that of [22]) does not use the semigroup property in an extensive way. Theorem 5.6 gives a result on the numerical range for a single operator $T$ instead of a semigroup. Furthermore, one could state theorem 5.6 for an operator acting on $L^{p}$ for a single value of $p$, by replacing $(\mathcal{P})$ by some condition for an operator $L^{p} \rightarrow L^{p}$.

In section 2, we introduce non-commutative $L^{p}$-spaces and mention their properties that we need and give some examples. In section 3, completely positive and completely bounded maps are developed as far as needed in the article. The diffusion semigroups are then defined in section 4 and a basic guiding example is discussed. Section 5 contains the main theorems, and sections 6 and 7 are devoted to examples of diffusion semigroups to which our method applies.

\section{Background on von Neumann algebras and NON-COMmutative $L^{p}$-SPACES}

Throughout the paper, we denote $M$ a von Neumann algebra (see e.g. [29] for the definition) and assume that there is a semifinite, normal, faithful (s.n.f.) trace $\tau$ on $M$. The following examples for $(M, \tau)$ will frequently occur.

Examples of von Neumann algebras and definitions.

1. For every $n \in \mathbb{N}$, we have the algebra of matrices $M_{n}=B\left(l_{n}^{2}\right)=\mathbb{C}^{n \times n}$, equipped with the common trace $\tau=\operatorname{tr}$. Note that every finite dimensional von Neumann algebra has a representation as a direct sum

$$
M=M_{n_{1}} \oplus \ldots \oplus M_{n_{K}}
$$


with $\tau\left(x_{1} \oplus \ldots \oplus x_{K}\right)=\sum_{k=1}^{K} \lambda_{k} \operatorname{tr}\left(x_{k}\right)$ for some $K \in \mathbb{N}$ and $\lambda_{j}>0$. Further,

$$
\left\|x_{1} \oplus \ldots \oplus x_{K}\right\|_{M}=\sup _{k=1}^{K}\left\|x_{k}\right\|_{M_{n_{k}}} .
$$

2. $M$ is called hyperfinite if there exists a net of finite dimensional $*$-subalgebras $A_{\alpha}$ which are directed by inclusion, such that $\bigcup A_{\alpha}$ is $w^{*}$-dense in $M$. If $\tau$ is a s.n.f. trace then one can always choose $A_{\alpha}$ such that $\left.\tau\right|_{A_{\alpha}}$ is finite [25, chap 3].

Let us show this under the additional assumption that the net can be chosen as a sequence $\left(A_{n}\right)_{n}$. This is the case in most examples. Let $\left(q_{k}\right)_{k}$ be a sequence of orthogonal projections in $M$ such that $\tau\left(q_{k}\right)<\infty, \operatorname{Im}\left(q_{k}\right) \subseteq \operatorname{Im}\left(q_{k+1}\right)$ and $q_{k} \rightarrow 1$ in the strong operator topology. Such a sequence exists since $\tau$ is semifinite. Put now $p_{k}=q_{k}-q_{k-1}$ and $A_{n}^{\prime}=\bigoplus_{k \leq n} p_{k} A_{n} p_{k}$. Then $\left(A_{n}^{\prime}\right)_{n}$ has the desired properties, i.e. $\left.\tau\right|_{A_{n}^{\prime}}$ is finite.

3. Let $(\Omega, \mu)$ be a $\sigma$-finite measure space. Then $M=L^{\infty}(\Omega)=L^{\infty}(\Omega, \mu)$ is a von Neumann algebra with the s.n.f. trace $\tau(f)=\int f d \mu$. $M$ is hyperfinite: Indeed, we explicitly give a net of finite dimensional $*$-subalgebras. We call a finite collection $\left\{A_{1}, \ldots, A_{n}\right\}$ of pairwise disjoint measurable subsets of $\Omega$ such that $0<\mu\left(A_{k}\right)<\infty$ a semi-partition. Let $\mathcal{A}$ be the set of all semi-partitions. $\mathcal{A}$ is directed by $\left\{A_{1}, \ldots, A_{n}\right\} \prec\left\{B_{1}, \ldots, B_{m}\right\}$ iff any $A_{k}$ is the union of some of the $B_{k}^{\prime} s$. For $\alpha=\left\{A_{1}, \ldots, A_{n}\right\} \in \mathcal{A}$, put $M_{\alpha}:=\left\{\sum_{k=1}^{n} c_{k} \chi_{A_{k}}: c_{k} \in \mathbb{C}\right\} \subset M$. Clearly, $\left.\tau\right|_{M_{\alpha}}$ is finite and for any $x \in L^{\infty}(\Omega)$ and $y \in L^{1}(\Omega), \int x_{\alpha} y d \mu \rightarrow \int x y d \mu$, which is the $w^{*}$-density.

4. If $M \subset B(H)$ and $N \subset B(K)$ is a further von Neumann algebra with s.n.f. trace $\sigma$, then $N \bar{\otimes} M$ defined as the $w^{*}$-closure of $N \otimes M$ in $B\left(K \otimes_{2} H\right)$ is again a von Neumann algebra. $(\sigma \otimes \tau)(x \otimes y):=\sigma(x) \tau(y)$ can be extended to a s.n.f. trace on $N \bar{\otimes} M$. We will use this fact for the cases $N=M_{n}$ as in 1 and $N=L^{\infty}(\Omega)$ as in 3. $L^{\infty}(\Omega) \bar{\otimes} M$ can be naturally identified with the space of $w^{*}$-measurable, essentially bounded functions $\Omega \rightarrow M$, see [3, p. 40-41].

For $1 \leq p<\infty$, the non-commutative $L^{p}$-spaces $L^{p}(M)=L^{p}(M, \tau)$ are defined as follows. If $S_{+}$is the set of all positive $x \in M$ (i.e. $x=x^{*}$ and $\sigma(x) \subset[0, \infty)$ ) such that $\tau(x)<\infty$ and $S$ is its linear span, then $L^{p}(M)$ is the completion of $S$ with respect to the norm $\|x\|_{p}=\tau\left(|x|^{p}\right)^{1 / p}$. It can also be described as a space of unbounded operators $x$ affiliated to $M$ in a certain sense such that $\tau\left(|x|^{p}\right)^{1 / p}<\infty$, where the domain of $\tau$ is extended to all of $L^{1}(M)$. One sets $L^{\infty}(M)=M$. As for the commutative (i.e. usual) $L^{p}$-spaces, one has: $L^{p}(M)^{\prime}=L^{q}(M)$ via the duality $(x, y) \mapsto \tau(x y)$, for $1 \leq p<\infty$ and $\frac{1}{p}+\frac{1}{q}=1$. We denote this duality from now on by $\langle x, y\rangle$. The Hölder inequality holds in the form $\|x\|_{L^{p}(M)}=\sup \left\{|\langle x, y\rangle|:\|y\|_{L^{q}(M)} \leq 1\right\}$. The space $L^{2}(M)$ is a Hilbert space with respect to the scalar product $(x, y) \mapsto\left\langle x, y^{*}\right\rangle$. For $1 \leq p, q \leq \infty,\left(L^{p}(M), L^{q}(M)\right)$ is, in the sense of complex interpolation [2], a compatible couple of spaces such that $\left(L^{p}(M), L^{q}(M)\right)_{\theta}=L^{r}(M)$ with $\frac{1}{r}=\frac{\theta}{q}+\frac{1-\theta}{p}$.

See $[30,27]$ for further reference on non-commutative $L^{p}$-spaces. Examples which will appear are:

Examples of non-commutative $L^{p}$-spaces.

1. For $(M, \tau)=\left(M_{n}, \operatorname{tr}\right)$, we write $S_{n}^{p}=L^{p}\left(M_{n}\right)$. More generally, if $H$ is a Hilbert space and $\operatorname{tr}$ the usual trace on $B(H)$, then $S^{p}(H)=L^{p}\left(B(H)\right.$, tr). If $H=l^{2}$, then we write 
$S^{p}=S^{p}(H)$.

2. If $M$ is finite dimensional and $(M, \tau)=\left(M_{n_{1}}, \lambda_{1} \operatorname{tr}\right) \oplus \ldots \oplus\left(M_{n_{K}}, \lambda_{K} \operatorname{tr}\right)$, then for $x=$ $x_{1} \oplus \ldots \oplus x_{K},\|x\|_{L^{p}(M)}^{p}=\sum_{k} \lambda_{k}\left\|x_{k}\right\|_{S_{n}^{p}}^{p}$.

3. If $(\Omega, \mu)$ is a $\sigma$-finite measure space and $M=L^{\infty}(\Omega)$, then $L^{p}(\Omega)=L^{p}(M), 1 \leq p \leq \infty$.

4. If $M=L^{\infty}(\Omega)$ and $N$ is a further von Neumann algebra with s.n.f. trace $\sigma$, then $L^{p}(M \bar{\otimes} N)$ is naturally isometric to the Bochner space $L^{p}\left(\Omega, L^{p}(N)\right)$ for $1 \leq p<\infty$.

Finally, the following notion of a dual element will play an eminent role.

Definition 2.1. Let $1<p<\infty$ and $q=\frac{p}{p-1}$ the conjugate number. Let $x \in L^{p}(M)$. Then $x$ has a polar decomposition $x=u|x|$ with $u \in M$ unitary and $|x|=\left(x^{*} x\right)^{1 / 2}$. The dual element of $x$ is defined as $\widehat{x}=|x|^{p-1} u^{*}$.

Lemma 2.2. The above defined $\widehat{x}$ is the unique element in $L^{q}(M)$ with:

(1) $\langle x, \widehat{x}\rangle=\|x\|_{p}^{p}$.

(2) $\|x\|_{p}^{p}=\|\widehat{x}\|_{q}^{q}$.

Further, the (in non trivial cases nonlinear) mapping

$$
\hat{:} \begin{cases}L^{p}(M) & \longrightarrow L^{q}(M) \\ x & \longmapsto \widehat{x}\end{cases}
$$

is norm-continuous.

Proof. It is plain that $\widehat{x}$ satisfies the claimed properties. On the other hand, it is well known that $L^{p}(M)$ is uniformly smooth, which implies uniqueness. To see the continuity of $x \mapsto \widehat{x}$, let $x, x_{1}, x_{2}, \ldots \in L^{p}(M)$ such that $x_{n} \rightarrow x$. We can exclude the trivial case $x=0$. Since $\left\|\widehat{x_{n}}\right\|_{q}=\left\|x_{n}\right\|_{p}^{p / q}$ is bounded, the Banach-Alaoglu theorem gives a weak limit point of $\left(\widehat{x_{n}}\right)_{n}$. We show that any such limit point $y$ equals $\widehat{x}$, which implies that $\widehat{x}$ is the weak limit of $\widehat{x_{n}}$. Since $L^{p}(M)$ is uniformly convex and $\left\|\widehat{x_{n}}\right\|_{q} \rightarrow\|\widehat{x}\|_{q}$, it will follow that $\left\|\widehat{x_{n}}-\widehat{x}\right\|_{q} \rightarrow 0$.

Let $y=\mathrm{w}-\lim _{k} \widehat{x_{n_{k}}}$. We have

$$
\langle x, y\rangle=\lim _{k}\left\langle x, \widehat{x_{n_{k}}}\right\rangle=\lim _{k}\left\langle x_{n_{k}}, \widehat{x_{n_{k}}}\right\rangle+\left\langle x-x_{n_{k}}, \widehat{x_{n_{k}}}\right\rangle=\|x\|_{p}^{p}+0 .
$$

This shows that $y$ satisfies (1) of the lemma, and that $\|y\|_{q} \geq\|x\|_{p}^{p-1}$. On the other hand, $\|y\|_{q} \leq \lim \sup _{k}\left\|\widehat{x_{n_{k}}}\right\|_{q}=\|\widehat{x}\|_{q}=\|x\|_{p}^{p-1}$, so that $y$ satisfies (2). By uniqueness of $\widehat{x}, y=$ $\widehat{x}$.

\section{Operators BetWeEn NON-COMMUTATIVE $L^{p}$-SPACES}

For $n \in \mathbb{N}$ and $1 \leq p \leq \infty$, we denote by $S_{n}^{p}\left(L^{p}(M)\right)$ the space $S_{n}^{p} \otimes L^{p}(M)=\left\{\left(x_{i j}\right)_{i j}\right.$ : $\left.i, j=1, \ldots, n, x_{i j} \in L^{p}(M)\right\}$ equipped with the norm of $L^{p}\left(M_{n} \otimes M, \operatorname{tr} \otimes \tau\right)$ [25, chap 1]. Let $T: L^{p}(M) \rightarrow L^{p}(N)$ be a linear mapping, where $N$ is a further von Neumann algebra with s.n.f. trace $\sigma$. Following [25, lem 1.7], we call $T$ completely bounded if

$$
\|T\|_{c b}:=\sup _{n}\left\|I_{S_{n}^{p}} \otimes T:\left(x_{i j}\right)_{i j} \mapsto\left(T x_{i j}\right)_{i j}\right\|_{B\left(S_{n}^{p}\left(L^{p}(M)\right), S_{n}^{p}\left(L^{p}(N)\right)\right)}<\infty,
$$

and completely contractive if this quantity is less than 1 . Clearly, $\|T\| \leq\|T\|_{c b}$. If $T$ : $L^{p}(M) \rightarrow L^{p}(N)$ is completely bounded for $p=1$ and $p=\infty$, then by complex interpolation,

$$
\left\|T: L^{q}(M) \rightarrow L^{q}(N)\right\|_{c b} \leq\left\|T: L^{1}(M) \rightarrow L^{1}(N)\right\|_{c b}^{1 / q}\|T: M \rightarrow N\|_{c b}^{1-1 / q}
$$


for any $1<q<\infty$. $T: L^{p}(M) \rightarrow L^{p}(N)$ is called positive if $T x \geq 0$ for all $x \geq 0$. $T$ is called completely positive, if $I_{S_{n}^{p}} \otimes T \in B\left(S_{n}^{p}\left(L^{p}(M)\right), S_{n}^{p}\left(L^{p}(N)\right)\right)=B\left(L^{p}\left(M_{n} \otimes M\right), L^{p}\left(M_{n} \otimes N\right)\right)$ is positive for all $n \in \mathbb{N}$. In the case $M=N=M_{n}$, Choi showed in [4] the following characterization:

$$
T: M_{n} \rightarrow M_{n} \text { completely positive } \Longleftrightarrow \exists a_{1}, \ldots, a_{N} \in M_{n}: T x=\sum_{k=1}^{N} a_{k}^{*} x a_{k} .
$$

Assume that $\left.T \in B\left(L^{p}(M), L^{p}(N)\right)\right)$ for some $1 \leq p \leq \infty$ and that $T$ is $w^{*}$-continuous if $p=\infty$. In view of the duality $L^{p}(M)^{\prime}=L^{q}(M)$ for $1 \leq p<\infty, q=\frac{p}{p-1}$, the operator $T^{\prime}: L^{q}(N) \rightarrow L^{q}(M)$ is defined. If $p=\infty$, we denote $T^{\prime}: L^{1}(M) \rightarrow L^{1}(M)$ the pre-adjoint operator.

Lemma 3.1. Let $N, \sigma, p, q, T$ be as above.

(1) If $T: L^{p}(M) \rightarrow L^{p}(N)$ is (completely) positive, then $T^{\prime}: L^{q}(N) \rightarrow L^{q}(M)$ is (completely) positive also.

(2) If $T$ is completely bounded, then $T^{\prime}$ is also completely bounded, with the same cbnorm.

Proof. $T$ is positive if and only if $\sigma((T a) b) \geq 0$ for all positive $a \in L^{p}(M)$ and $b \in L^{q}(N)$. On the other hand, $\sigma((T a) b)=\tau\left(a\left(T^{\prime} b\right)\right)$, so that the positivity part follows. The complete positivity part is then a consequence of $\left(I_{S_{n}^{p}} \otimes T\right)^{\prime}=I_{S_{n}^{q}} \otimes T^{\prime}$. This also gives the complete boundedness statement, in view of (3.1).

\section{Non-COMmUtative DifFUSION SEMIGROUPS}

Let $T: M \rightarrow M$ be a $w^{*}$-continuous operator with $\|T\|_{M \rightarrow M} \leq 1$. Assume that

$$
\text { for } x, y \in M \cap L^{1}(M),\left\langle T x, y^{*}\right\rangle=\left\langle x,(T y)^{*}\right\rangle \text {. }
$$

We call a $T$ with this property self-adjoint. Then by the Hölder inequality, $\left.T\right|_{M \cap L^{1}(M)}$ extends to a contraction $T_{1}: L^{1}(M) \rightarrow L^{1}(M)$ and by complex interpolation, also to $T_{p}$ with

$$
\left\|T_{p}: L^{p}(M) \rightarrow L^{p}(M)\right\| \leq 1 \quad(1 \leq p \leq \infty) .
$$

Since $T$ is $w^{*}$-continuous, (4.1) yields that $T_{1}=T^{\prime}\left(\cdot^{*}\right)^{*}$. Clearly, $T_{2}: L^{2}(M) \rightarrow L^{2}(M)$ is self-adjoint in the classical sense. If $T: M \rightarrow M$ is in addition completely contractive, then by lemma 3.1, $T_{1}=T^{\prime}\left(\cdot^{*}\right)^{*}: L^{1}(M) \rightarrow L^{1}(M)$ is also completely contractive, and hence $T_{p}$ also.

The following notion of a (non-commutative) diffusion semigroup has been defined in [16] and generalizes Stein's setting in [28].

Definition 4.1. Let $\left(T_{t}\right)_{t \geq 0}$ be a family of completely contractive operators of the above type. $\left(T_{t}\right)$ is called a diffusion semigroup (on $M$ ) if

$$
\begin{aligned}
& T_{0}=I_{M} \text { and } T_{t} T_{s}=T_{t+s} \text { for } t, s \geq 0 . \\
& T_{t} x \rightarrow x \text { as } t \rightarrow 0 \text { in the } w^{*} \text { topology. }
\end{aligned}
$$


Clearly, for $1 \leq p<\infty,\left(T_{t, p}\right)$ is a semigroup on $L^{p}(M)$ and by [8, prop 1.23], (4.3) implies that $\left(T_{t, p}\right)$ is strongly continuous. Examples of such diffusion semigroups are given in [16, chap $8,9,10$ ] and will be discussed in section 6 .

It is shown in [16, chap 5] - using the functional calculus for self-adjoint operators and a version of Stein's complex interpolation - that for $\left(T_{t, p}\right)$, there exists an analytic and contractive extension to a sector $S\left(\frac{\pi}{2}-\pi\left|\frac{1}{p}-\frac{1}{2}\right|\right)$, where we put

$$
S(\omega)=\{z \in \mathbb{C} \backslash\{0\}:|\arg z|<\omega\} .
$$

This means that there exists an analytic function $S\left(\frac{\pi}{2}-\pi\left|\frac{1}{p}-\frac{1}{2}\right|\right) \rightarrow B\left(L^{p}(M)\right), z \mapsto S_{z}$ such that $S_{t}=T_{t, p}$ for $t>0$ and $\left\|S_{z}\right\|_{B\left(L^{p}(M)\right)} \leq 1$. The major question of this article is:

Given a diffusion semigroup $\left(T_{t}\right)$ on $M$ and $1<p<\infty$, what is the optimal $\omega_{p}>0$ such that $T_{t, p}$ has an analytic and contractive extension to $S\left(\omega_{p}\right)$ ?

This question and related ones have been studied in the commutative case in $[1,22,23$, $31,17,18,5,6]$.

In the rest of this section, let us work out the candidate for $\omega_{p}$. First recall the following characterization.

Proposition 4.2. Let $\left(T_{t, p}\right)$ be a $c_{0}$-semigroup on $L^{p}(M)$ for some $1<p<\infty$. Denote $A_{p}$ its generator. Fix some $\omega \in\left(0, \frac{\pi}{2}\right)$. Then the following are equivalent.

(1) $-\left\langle A_{p} x, \widehat{x}\right\rangle \in \overline{S\left(\frac{\pi}{2}-\omega\right)}$ for all $x \in D\left(A_{p}\right)$.

(2) $\left(T_{t, p}\right)$ has an analytic and contractive extension to $S(\omega)$.

The first condition is obviously verified if

$$
\left\langle\left(I-T_{t, p}\right) x, \widehat{x}\right\rangle \in \overline{S\left(\frac{\pi}{2}-\omega\right)} \text { for all } x \in L^{p}(M) \text { and } t>0 .
$$

Proof. See for example [10, thm 5.9]

The following easy example already gives a good insight into what we can expect.

Example 4.3. Let $M$ be the commutative 2-dimensional von Neumann algebra $l_{2}^{\infty}$ with trace $\tau((a, b))=a+b$. We consider $T_{t}=e^{t A}$ with

$$
A=\left(\begin{array}{cc}
-1 & 1 \\
1 & -1
\end{array}\right)=-e \otimes e
$$

where $e=(1,-1)$. Then $A^{n}=-2 A^{n-1}=(-2)^{n-1} A$ for $n \geq 2$. Hence

$$
T_{t}=I_{M}-\frac{1}{2} \sum_{n=1}^{\infty} \frac{(-2 t)^{n}}{n !} A=\frac{1}{2}\left(\begin{array}{cc}
1+e^{-2 t} & 1-e^{-2 t} \\
1-e^{-2 t} & 1+e^{-2 t}
\end{array}\right) .
$$

Since this matrix is self-adjoint and $\left|1+\frac{1}{2} e^{-2 t}\right|+\left|1-\frac{1}{2} e^{-2 t}\right|=1$ for all $t \geq 0,\left(T_{t}\right)$ is indeed a diffusion semigroup. Now fix some $1<p<\infty$ and let $x=(a, b) \in l_{2}^{p}$. Then $\widehat{x}=(\widehat{a}, \widehat{b})=\left(\bar{a}|a|^{p-2}, \bar{b}|b|^{p-2}\right)$ and

$$
-\langle A x, \widehat{x}\rangle=(a-b)(\widehat{a}-\widehat{b}) .
$$


To answer our angle question, in view of the preceding proposition, we are supposed to determine the smallest sector containing this quantity for arbitrary $a, b \in \mathbb{C}$. The solution is the following proposition which appears in [22, lem 2.2].

Proposition 4.4. Let $1<p<\infty, a, b \in \mathbb{C}$. Then for $\omega_{p}=\arctan \frac{|p-2|}{2 \sqrt{p-1}}$,

$$
(a-b)(\widehat{a}-\widehat{b})=|a|^{p}+|b|^{p}-a \bar{b}|b|^{p-2}-b \bar{a}|a|^{p-2} \in \overline{S\left(\omega_{p}\right)} .
$$

Further, this result is optimal, i.e. the statement is false for any $\omega<\omega_{p}$.

Proof. The fact that $z=(a-b)(\widehat{a}-\widehat{b}) \in \overline{\Sigma\left(\omega_{p}\right)}$ has been shown in [22, lem 2.2]. We show the optimality for the convenience of the reader. Let $b=1$ and $a=r e^{i \phi}$ with $r \neq 1$. Then $z=r^{p}+1-r e^{i \phi}-r^{p-1} e^{-i \phi}$, so that

$$
\begin{aligned}
& \operatorname{Im} z=-r \sin \phi+r^{p-1} \sin \phi, \\
& \operatorname{Re} z=r^{p}+1-r \cos \phi-r^{p-1} \cos \phi,
\end{aligned}
$$

whence

$$
\left(\frac{\operatorname{Im} z}{\operatorname{Re} z}\right)^{2}=\frac{\left(r^{p-1}-r\right)^{2}\left(1-\cos ^{2} \phi\right)}{\left(r^{p}+1-r \cos \phi-r^{p-1} \cos \phi\right)^{2}} .
$$

Maximizing this expression in $\phi$, i.e. choosing $\cos \phi=\frac{r\left(r^{p-2}+1\right)}{\left(r^{p}+1\right)^{2}}<1$ gives

$$
\left(\frac{\operatorname{Im} z}{\operatorname{Re} z}\right)^{2}=\frac{r^{2}\left(r^{p-2}-1\right)}{\left(r^{2}-1\right)\left(r^{2 p-2}-1\right)} .
$$

The limit for $r \rightarrow 1$ of this expression is $\frac{(p-2)^{2}}{4(p-1)}$, so that $|\arg z| \rightarrow \frac{|p-2|}{2 \sqrt{p-1}}$.

From now on, write

$$
\Sigma_{p}=\overline{S\left(\arctan \frac{|p-2|}{2 \sqrt{p-1}}\right)}, \quad \Sigma_{p}^{\prime}=S\left(\frac{\pi}{2}-\arctan \frac{|p-2|}{2 \sqrt{p-1}}\right) .
$$

In view of the above, $\Sigma_{p}$ is our candidate for the sector which supports the numerical range of $-A_{p}$, and we are looking for diffusion semigroups $\left(T_{t}\right)$ such that:

For every $1<p<\infty, T_{t, p}$ has an analytic and contractive extension $\Sigma_{p}^{\prime} \rightarrow B\left(L^{p}(M)\right)$.

\section{The Angle Theorem}

We begin with some notation. If $A, B, C, D \in B\left(L^{p}(M)\right)$, we denote

$$
\left(\begin{array}{cc}
A & B \\
C & D
\end{array}\right): L^{p}\left(M_{2} \otimes M\right) \rightarrow L^{p}\left(M_{2} \otimes M\right),\left(\begin{array}{cc}
a & b \\
c & d
\end{array}\right) \mapsto\left(\begin{array}{cc}
A(a) & B(b) \\
C(c) & D(d)
\end{array}\right) .
$$

For $p=2$, this operator is self-adjoint if and only if $A, B, C, D$ are all self-adjoint.

The key notion to establish the theorem for the analytic extension of a diffusion semigroup is the following one. 
Definition 5.1. Let $T: M \rightarrow M$ be a $w^{*}$-continuous operator. Denote $T_{*}: M \rightarrow$ $M, T_{*}(x)=T\left(x^{*}\right)^{*}$. Then we say that $T$ satisfies $(\mathcal{P})$ if there exist $S_{1}, S_{2}: M \rightarrow M$ such that

$$
W:=\left(\begin{array}{cc}
S_{1} & T \\
T_{*} & S_{2}
\end{array}\right): M_{2} \otimes M \rightarrow M_{2} \otimes M
$$

is completely positive, completely contractive and self-adjoint.

Note that a completely positive linear mapping between von Neumann algebras is completely contractive iff the image of the unity has norm less than 1 [24, prop 3.6].

Hence we can replace the complete contractivity in definition 5.1 by the assumption

$$
\left\|W\left(\begin{array}{ll}
1 & 0 \\
0 & 1
\end{array}\right)\right\| \leq 1
$$

Remark 5.2. 1. A $T$ satisfying $(\mathcal{P})$ is necessarily completely contractive and self-adjoint. Indeed, $W$ is self-adjoint iff $S_{1}, S_{2}$ and $T$ are. Further, it is well-known that the complete positivity of $W$ implies that $T$ is completely contractive.

2. On the other hand, if $T$ is completely contractive, self-adjoint and in addition completely positive, then it satisfies $(\mathcal{P})$. Just take $S_{1}=S_{2}=T$, and note that $T_{*}=T$. Then $W=\left(\begin{array}{cc}T & T \\ T & T\end{array}\right)$ is again completely positive, completely contractive on $M_{2}(M)$ and selfadjoint.

3. Assume that $(M, \tau)=\left(M_{n}\right.$, tr $)$. It is well-known that $T: M_{n} \rightarrow M_{n}$ is completely contractive if and only if there exist $a_{1}, \ldots, a_{N}$ and $b_{1}, \ldots, b_{N}$ such that $T x=\sum_{k=1}^{N} a_{k} x b_{k}$ and

$$
\left\|\sum_{k=1}^{N} a_{k} a_{k}^{*}\right\| \leq 1, \quad\left\|\sum_{k=1}^{N} b_{k}^{*} b_{k}\right\| \leq 1
$$

$T: x \mapsto \sum_{k} a_{k} x b_{k}$ is self-adjoint if and only if $\sum_{k} a_{k} x b_{k}=\sum_{k} a_{k}^{*} x b_{k}^{*}$ for all $x$. On the other hand, $T$ satisfies $(\mathcal{P})$ if and only if

$$
\exists a_{1}, \ldots, a_{N}, b_{1}, \ldots, b_{N} \in M_{n} \text { self-adjoint : } T x=\sum_{k=1}^{N} a_{k} x b_{k}, \sum_{k} a_{k}^{2} \leq 1, \sum_{k} b_{k}^{2} \leq 1 .
$$

Indeed, if (5.2) is satisfied, then put $S_{1} x=\sum_{k} a_{k} x a_{k}$ and $S_{2} x=\sum_{k} b_{k} x b_{k}$. Then

$$
\left(\begin{array}{ll}
S_{1} & T \\
T_{*} & S_{2}
\end{array}\right) x=\sum_{k} c_{k} x c_{k}
$$

with $c_{k}=\left(\begin{array}{cc}a_{k} & 0 \\ 0 & b_{k}\end{array}\right)$. Property $(\mathcal{P})$ follows, since $c_{k}$ is self-adjoint and $\sum c_{k}^{2} \leq 1$.

Conversely, if $(\mathcal{P})$ is satisfied, then by the complete positivity of $W$ and (3.2), there exist $c_{1}, \ldots, c_{N} \in M_{2 n}$ such that $W x=\sum_{k} c_{k}^{*} x c_{k}$. Since $W$ is self-adjoint, $\sum_{k} c_{k}^{*} x c_{k}=\sum_{k} c_{k} x c_{k}^{*}$ 
and consequently,

$$
W x=\sum_{k}\left(\frac{c_{k}+c_{k}^{*}}{2}\right) x\left(\frac{c_{k}+c_{k}^{*}}{2}\right)+\sum_{k}\left(\frac{c_{k}-c_{k}^{*}}{2 i}\right) x\left(\frac{c_{k}-c_{k}^{*}}{2 i}\right) .
$$

We have $\|W(1)\| \leq 1$, so replacing $\left(c_{1}, \ldots, c_{N}\right)$ by $\left(\left(c_{1}+c_{1}^{*}\right) / 2, \ldots,\left(c_{N}+c_{N}^{*}\right) / 2,\left(c_{1}-\right.\right.$ $\left.\left.c_{1}^{*}\right) /(2 i), \ldots,\left(c_{N}-c_{N}^{*}\right) /(2 i)\right)$, one can assume that the $c_{k}^{\prime} s$ are self-adjoint and $\left\|\sum_{k} c_{k}^{2}\right\| \leq 1$. Write $c_{k}=\left(\begin{array}{ll}a_{k} & d_{k} \\ d_{k}^{*} & b_{k}\end{array}\right)$. By definition of $W, T x=\sum_{k} a_{k} x b_{k}$. Further, $\left\|\sum_{k} a_{k}^{2}\right\|,\left\|\sum_{k} b_{k}^{2}\right\| \leq$ $\left\|\sum_{k} c_{k}^{2}\right\|$, so that $a_{k}, b_{k}$ match (5.2).

4. The property $(\mathcal{P})$ is connected to the definition of decomposable maps. $T: M \rightarrow M$ is by definition decomposable $\left(\|T\|_{d e c} \leq 1\right)$ if $S_{1}$ and $S_{2}$ exist such that $W=\left(\begin{array}{cc}S_{1} & T \\ T_{*} & S_{2}\end{array}\right)$ is completely positive (and contractive) [26, p. 130]. One has $\|T\|_{\text {dec }} \leq 1$ for all complete contractions $T: M \rightarrow M$ iff $M$ is hyperfinite [12]. In general, the assumptions $\|T\|_{\text {dec }} \leq 1$ and $T$ self-adjoint do not imply $(\mathcal{P})$, see the example below. However, we will see in section 6 that this holds true in some special cases.

Example 5.3. Parts 1 and 2 of the preceding remark lead to the question if the property $(\mathcal{P})$ is equivalent to complete contractivity and self-adjointness. But in general, $(\mathcal{P})$ is strictly stronger. Indeed, there is a self-adjoint and complete contractive $T$ which does not satisfy $(\mathcal{P})$. The author is grateful to Éric Ricard for showing him the following example. The operator space theory used here goes beyond what is explained in section 2 , see for example $[26,9]$.

Let $n \in \mathbb{N}$ and $\left(E_{i j}\right)_{0 \leq i, j \leq n}$ be the canonical basis of $M_{n+1}$. Define $T: M_{n+1} \rightarrow M_{n+1}$ by

$$
T x=\sum_{i=1}^{n} E_{i 0} x E_{i 0}+\sum_{i=1}^{n} E_{0 i} x E_{0 i} .
$$

Then $T$ is self-adjoint and by writing

$$
T x=\sum_{i=1}^{n}\left(n^{1 / 4} E_{i 0}\right) x\left(n^{-1 / 4} E_{i 0}\right)+\sum_{i=1}^{n}\left(n^{-1 / 4} E_{0 i}\right) x\left(n^{1 / 4} E_{0 i}\right),
$$

one sees that $\|T\|_{c b} \leq \sqrt{n}$ (cf. 5.2 .3 above). Now assume that $a_{1}, \ldots, a_{N}, b_{1}, \ldots, b_{N} \in M_{n+1}$ are self-adjoint such that $T x=\sum_{k=1}^{N} a_{k} x b_{k}$. We will show that

$$
\left\|\sum_{k} a_{k}^{2}\right\|^{1 / 2}\left\|\sum_{k} b_{k}^{2}\right\|^{1 / 2} \geq n
$$

so that for $n \geq 2$, the self-adjoint completely contractive operator $\frac{1}{\sqrt{n}} T$ does not satisfy $(\mathcal{P})$.

We denote $R_{N}$ and $C_{N}$ the row and column operator space of dimension $N$ [26, p. 21]. Further, $R_{N} \cap C_{N}$ is equipped with the operator space structure

$$
\left\|\left(x_{i j}\right)\right\|=\max \left\{\left\|\left(x_{i j}\right)\right\|_{M_{n}\left(R_{N}\right)},\left\|\left(x_{i j}\right)\right\|_{M_{n}\left(C_{N}\right)}\right\}
$$


and $R_{N}+C_{N}$ is the operator space dual of $R_{N} \cap C_{N}$ [26, p. 55,194]. Then for any operator space $X$ and any $x_{1}, \ldots, x_{N} \in X$,

$$
\left\|\sum_{k=1}^{N} e_{k} \otimes x_{k}: X^{*} \rightarrow R_{N} \cap C_{N}\right\|_{c b}=\max \left\{\left\|\sum_{k} x_{k} x_{k}^{*}\right\|^{1 / 2},\left\|\sum_{k} x_{k}^{*} x_{k}\right\|^{1 / 2}\right\} .
$$

Let

$$
\alpha=\sum_{k=1}^{N} a_{k} \otimes e_{k}: l_{N}^{2} \rightarrow M_{n+1}, \quad \beta=\sum_{k=1}^{N} e_{k} \otimes b_{k}: S_{n+1}^{1} \rightarrow l_{N}^{2} .
$$

Here, $\left(e_{k}\right)_{1 \leq k \leq N}$ is the canonical basis of $l_{N}^{2}, a_{k} \otimes e_{k}$ maps $x$ to $\left\langle x, e_{k}\right\rangle_{l_{N}^{2}} a_{k}$ and $e_{k} \otimes b_{k}$ maps $x$ to $\operatorname{tr}\left(x b_{k}\right) e_{k}$. Then

$$
\begin{aligned}
\left\|\alpha: R_{N}+C_{N} \rightarrow M_{n+1}\right\|_{c b} & =\left\|\sum_{k} a_{k}^{2}\right\|^{1 / 2}, \\
\left\|\beta: S_{n+1}^{1} \rightarrow R_{N} \cap C_{N}\right\|_{c b} & =\left\|\sum_{k} b_{k}^{2}\right\|^{1 / 2} \text { and } \\
\alpha \beta x & =T x \text { for any } x \in M_{n+1} .
\end{aligned}
$$

Let us denote $C_{n} \oplus_{\infty} R_{n} \subset M_{n+1}$ the subspace spanned by $\left\{E_{i 0}, E_{0 i}: 1 \leq i \leq n\right\}$. In the same manner, we regard this space as $R_{n} \oplus_{1} C_{n} \subset S_{n+1}^{1}$. If $J: R_{n} \oplus_{1} C_{n} \rightarrow \bar{C}_{n} \oplus_{\infty} R_{n}$ is the identity, then $\alpha \beta$ is obtained by projecting canonically $S_{n+1}^{1}$ to $R_{n} \oplus_{1} C_{n}$, then applying $J$ and finally injecting $C_{n} \oplus_{\infty} R_{n}$ into $M_{n+1}$. Denote $\hat{\alpha}=p \alpha$ and $\hat{\beta}=\beta j$, where $p$ is the natural projection of $M_{n+1}$ onto $C_{n} \subset C_{n} \oplus_{\infty} R_{n} \subset M_{n+1}$ and $j$ is the embedding of $R_{n} \subset R_{n} \oplus_{1} C_{n} \subset S_{n+1}^{1}$ into $S_{n+1}^{1}$. Then one obtains the following commuting diagram

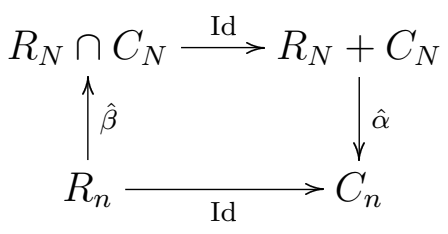

with

$$
\begin{gathered}
\left\|\hat{\alpha}: R_{N}+C_{N} \rightarrow C_{n}\right\|_{c b} \leq\left\|\sum_{k} a_{k}^{2}\right\|^{1 / 2}, \\
\left\|\hat{\beta}: R_{n} \rightarrow R_{N} \cap C_{N}\right\|_{c b} \leq\left\|\sum_{k} b_{k}^{2}\right\|^{1 / 2} .
\end{gathered}
$$

According to the factorization $I_{l_{N}^{2}}=\hat{\alpha} \hat{\beta}$ we have $n \leq\|\hat{\beta}\|_{H S}\|\hat{\alpha}\|_{H S}$. But the Hilbert-Schmidt norm of any $\gamma: l_{m_{1}}^{2} \rightarrow l_{m_{2}}^{2}$ equals the cb-norm of $\gamma: R_{m_{1}} \rightarrow C_{m_{2}}[26$, p. 21], so

$$
\begin{aligned}
n & \leq\left\|\hat{\beta}: R_{n} \rightarrow C_{N}\right\|_{c b}\left\|\hat{\alpha}: R_{N} \rightarrow C_{n}\right\|_{c b} \\
& \leq\left\|\hat{\beta}: R_{n} \rightarrow C_{N} \cap R_{N}\right\|_{c b}\left\|\hat{\alpha}: R_{N}+C_{N} \rightarrow C_{n}\right\|_{c b} \\
& \leq\left\|\sum_{k} b_{k}^{2}\right\|^{1 / 2}\left\|\sum_{k} a_{k}^{2}\right\|^{1 / 2} .
\end{aligned}
$$

This shows (5.3). 
Now the matrix version of the main theorem reads as follows.

Theorem 5.4. Let $n \in \mathbb{N}$ and $T: M_{n} \rightarrow M_{n}$ satisfy $(\mathcal{P})$. Fix some $p \in(1, \infty)$. Then for any $x \in S_{n}^{p}$,

$$
\langle(I-T) x, \widehat{x}\rangle \in \Sigma_{p}
$$

Proof. Use remark 5.2 and write $T x=\sum_{k=1}^{m} a_{k} x b_{k}$ with $a_{k}, b_{k}$ as in (5.2). Decompose

$$
x=u d v,
$$

with $u, v \in M_{n}$ unitaries and $d$ a diagonal matrix with non-negative diagonal entries $d_{1}, \ldots, d_{n}$. Then $\widehat{x}=v^{*} d^{p-1} u^{*}$. For simplifying the calculation, we write $g_{k}=u^{*} a_{k} u$ and $h_{k}=v b_{k} v^{*}$.

$$
\begin{aligned}
\langle(I-T) x, \widehat{x}\rangle & =\operatorname{tr}\left(d^{p}-\sum_{k} g_{k} d h_{k} d^{p-1}\right) \\
& =\sum_{r=1}^{n} d_{r}^{p}-\sum_{k, r, s} g_{k, r s} d_{s} h_{k, s r} d_{r}^{p-1} .
\end{aligned}
$$

Write $c_{r s}:=\sum_{k} g_{k, r s} h_{k, s r}$. Since $g_{k}$ and $h_{k}$ are self-adjoint, $c_{r s}=\overline{c_{s r}}$. Thus, the above expression equals

$$
\begin{aligned}
& \sum_{r} d_{r}^{p}-\frac{1}{2} \sum_{r, s} c_{r s} d_{s} d_{r}^{p-1}-\frac{1}{2} \sum_{r, s} \overline{c_{r s}} d_{r} d_{s}^{p-1} \\
= & \frac{1}{2}\left\{\sum_{r} d_{r}^{p}\left(1-\sum_{s}\left|c_{r s}\right|\right)+\sum_{s} d_{s}^{p}\left(1-\sum_{r}\left|c_{r s}\right|\right)+\sum_{r, s}\left(d_{r}^{p}\left|c_{r s}\right|+d_{s}^{p}\left|c_{r s}\right|-c_{r s} d_{s} d_{r}^{p-1}-\overline{c_{r s}} d_{r} d_{s}^{p-1}\right)\right\} .
\end{aligned}
$$

The expression in round brackets of the last double sum is a term $(a-b)(\widehat{a}-\widehat{b})$ as in proposition 4.4, putting

Since $\Sigma_{p}$ is closed under addition,

$$
a=d_{r}\left|c_{r s}\right|^{1 / p} \text { and } b=d_{s}\left|c_{r s}\right|^{1 / p} \frac{c_{r s}}{\left|c_{r s}\right|}
$$

$$
\sum_{r, s} d_{r}^{p}\left|c_{r s}\right|+d_{s}^{p}\left|c_{r s}\right|-c_{r s} d_{s} d_{r}^{p-1}-\overline{c_{r s}} d_{r} d_{s}^{p-1} \in \Sigma_{p}
$$

Moreover, it now suffices to show that

$$
1-\sum_{s}\left|c_{r s}\right| \geq 0,1-\sum_{r}\left|c_{r s}\right| \geq 0
$$

First we use Cauchy-Schwarz:

$$
\left(\sum_{s}\left|c_{r s}\right|\right)^{2}=\left(\sum_{s}\left|\sum_{k} g_{k, r s} h_{k, s r}\right|\right)^{2} \leq\left(\sum_{s} \sum_{k}\left|g_{k, r s}\right|^{2}\right)\left(\sum_{s} \sum_{k}\left|h_{k, s r}\right|^{2}\right) .
$$

We estimate the first factor:

$$
\sum_{s} \sum_{k}\left|\left(u^{*} a_{k} u\right)_{r s}\right|^{2}=\sum_{s} \sum_{k}\left(u^{*} a_{k} u\right)_{s r}\left(u^{*} a_{k} u\right)_{r s}=\sum_{k}\left(u^{*} a_{k}^{2} u\right)_{r r} \leq 1,
$$


where we use the assumption $\sum_{k} a_{k}^{2} \leq 1$ in the last inequality. In the same way, one estimates the second factor, which gives the first estimate in (5.4). The second estimate in (5.4) follows at once, since $\left|c_{r s}\right|=\left|c_{s r}\right|$.

Our next goal is to extend the theorem to hyperfinite von Neumann algebras instead of $M_{n}$ by a limit process. The following lemma contains the necessary information how the property $(\mathcal{P})$ and the dual element behave when passing from a "small" von Neumann algebra $N$ to a "big" von Neumann algebra $\mathcal{N}$ and vice versa.

Lemma 5.5. Let $(N, \sigma)$ and $(\mathcal{N}, \tilde{\sigma})$ be two von Neumann algebras with s.n.f. trace. Assume that there exist $J: N \rightarrow \mathcal{N}$ and $Q: \mathcal{N} \rightarrow N$ with the following properties:

(1) $J$ and $Q$ are completely positive,

(2) $J$ and $Q$ are (completely) contractive,

(3) $Q J=I_{N}$,

(4) $\langle J x, y\rangle=\langle x, Q y\rangle$ for all $x \in L^{1}(N) \cap N$ and $y \in L^{1}(\mathcal{N}) \cap \mathcal{N}$.

Then $J$ and $Q$ extend to complete contractions $J_{p}: L^{p}(N) \rightarrow L^{p}(\mathcal{N})$ and $Q_{p}: L^{p}(\mathcal{N}) \rightarrow$ $L^{p}(N)$ for any $1 \leq p \leq \infty$. Furthermore, the following holds.

(1) If $T: \mathcal{N} \rightarrow \mathcal{N}$ satisfies $(\mathcal{P})$, then also $Q T J: N \rightarrow N$ does. For all $1<p<\infty$ and $x \in L^{p}(N),\left\langle\left(I_{L^{p}(\mathcal{N})}-T_{p}\right) J_{p}(x), \widehat{J_{p}(x)}\right\rangle=\left\langle\left(I_{L^{p}(N)}-Q_{p} T_{p} J_{p}\right) x, \widehat{x}\right\rangle$.

(2) If $T: N \rightarrow N$ satisfies $(\mathcal{P})$, then also $J T Q: \mathcal{N} \rightarrow \mathcal{N}$ does. For all $1<p<\infty$ and $x \in L^{p}(N),\left\langle\left(I_{L^{p}(N)}-T_{p}\right) x, \widehat{x}\right\rangle=\left\langle\left(I_{L^{p}(\mathcal{N})}-J_{p} T_{p} Q_{p}\right) J_{p}(x), \widehat{J_{p}(x)}\right\rangle$.

Proof. The completely contractive extensions $J_{p}$ and $Q_{p}$ follow from assumption 4 by Hölder's inequality and complex interpolation, as in the beginning of section 4 .

(1) Let $W$ be an extension of $T$ according to the definition of $(\mathcal{P})$. Then $\widetilde{W}=\widetilde{Q} W \widetilde{J}$ is an appropriate extension of $Q T J$, where $\widetilde{Q}=I_{M_{2}} \otimes Q$ and $\widetilde{J}=I_{M_{2}} \otimes J$. Indeed, since $J$ and $Q$ are completely positive, also $\widetilde{J}$ and $\widetilde{Q}$ are, and therefore $\widetilde{W}$ is. As $J$ and $Q$ are completely contractive, $\widetilde{J}, \widetilde{Q}$, and thus $\widetilde{W}$ are contractive. By (5.1), $\widetilde{W}$ is completely contractive. It is plain to check the self-adjointness of $\widetilde{W}$. Just note that by the positivity of $\widetilde{Q}, \widetilde{Q}\left(x^{*}\right)=[\widetilde{Q}(x)]^{*}$, so $\widetilde{Q}_{2}$ is the adjoint of $\widetilde{J}_{2}$ in the Hilbert space sense.

For the second part, note that by approximation, $\left\langle Q_{p} x, y\right\rangle=\left\langle x, J_{q} y\right\rangle$ for any $x \in L^{p}(\mathcal{N}), y \in$ $L^{q}(N)$ and $1 \leq p, q \leq \infty$ conjugated exponents. Also assumption 3 extends to $Q_{p} J_{p}=I_{L^{p}(N)}$ for all $1 \leq p \leq \infty$. Now the assertion follows if we know that $\widehat{J_{p}(x)}=J_{q}(\widehat{x})$ for any $x \in L^{p}(N)$. We check the two determining properties of the dual element.

$$
\left\langle J_{q}(\widehat{x}), J_{p}(x)\right\rangle=\left\langle Q_{q} J_{q}(\widehat{x}), x\right\rangle=\langle\widehat{x}, x\rangle=\|x\|_{p}^{p} .
$$

Further,

$$
\left\|J_{q}(\widehat{x})\right\|_{q}^{q}=\|\widehat{x}\|_{q}^{q}=\|x\|_{p}^{p}=\left\|J_{p}(x)\right\|_{p}^{p}=\left\|\widehat{J_{p}(x)}\right\|_{q}^{q} .
$$

Here, we have used that $J_{q}$ (and $J_{p}$ ) is an isometry. This follows from $Q_{q} J_{q}=I_{L^{q}(N)}$ and the contractivity of $J_{q}$.

(2) Put $\widetilde{W}=\widetilde{J} W \widetilde{Q}$. The rest of the proof is very similar to that of (1). 
Theorem 5.6. Let $M$ be a hyperfinite von Neumann algebra and $T: M \rightarrow M$ satisfy $(\mathcal{P})$. Then for all $1<p<\infty$ and $x \in L^{p}(M)$,

$$
\left\langle\left(I_{L^{p}(M)}-T_{p}\right) x, \widehat{x}\right\rangle \in \Sigma_{p} .
$$

Proof. 1st case: $M$ finite dimensional. Then there exist $K \in \mathbb{N}, \lambda_{1}, \ldots, \lambda_{K}>0$ and $n_{1}, \ldots, n_{K} \in \mathbb{N}$ such that $(M, \tau)$ has a representation as a direct sum

$$
(M, \tau)=\left(M_{n_{1}}, \lambda_{1} \operatorname{tr}\right) \oplus \ldots \oplus\left(M_{n_{K}}, \lambda_{K} \operatorname{tr}\right) .
$$

We want to apply (2) of lemma 5.5.

Assume for a moment that $\lambda_{1}, \ldots, \lambda_{K} \in \mathbb{N}$. Take $N=M$ and $\mathcal{N}=M_{m}$ with $m=\sum_{k=1}^{K} \lambda_{k} n_{k}$, endowed with the standard trace tr. Put

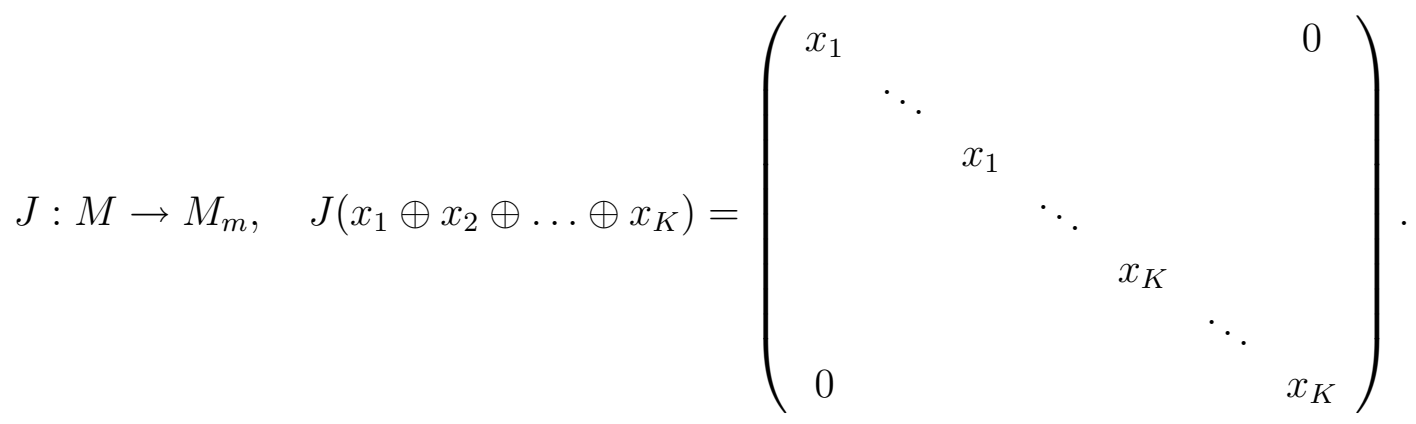

Here, the multiplicity of the $x_{k}^{\prime} s$ on the diagonal of the big matrix is $\lambda_{k}$. Let $Q: M_{m} \rightarrow M$ be defined by $\langle J x, y\rangle=\langle x, Q y\rangle$. $J$ is completely positive by its simple structure, and then $Q$ also is by lemma 3.1. $J$ is a contraction, since $\|J(x)\|=\max _{k}\left\|x_{k}\right\|=\|x\|$. $Q$ is a contraction, since $\|x\|_{L^{1}(M)}=\sum_{k} \lambda_{k}\left\|x_{k}\right\|_{S_{n_{k}}^{1}}=\|J(x)\|_{S_{m}^{1}}$. Finally, the identity $Q J=I_{M}$ is easy to check, so that the assumptions of lemma 5.5 are satisfied, and

$$
\left\langle\left(I_{L^{p}(M)}-T_{p}\right) x, \widehat{x}\right\rangle=\left\langle\left(I_{M_{m}}-J T Q\right) J x, \widehat{J x}\right\rangle \stackrel{\text { thm }}{\in}{ }^{5.4} \Sigma_{p} .
$$

Assume now that $\lambda_{k} \in \mathbb{Q}$. Then let $t \in \mathbb{N}$ be the common denominator of the $\lambda_{k}^{\prime} s$. Put $m=t \sum_{k} \lambda_{k} n_{k}$ and $\mathcal{N}=M_{m}$ with the trace $t^{-1} \cdot \operatorname{tr}$. Use the same $J$ as before, the multiplicity of the $a_{k}^{\prime} s$ being now $t \lambda_{k}$. We appeal again to lemma $5.5(2)$. Note that the theorem 5.4 is also valid with the modified trace $t^{-1} \cdot \operatorname{tr}$.

The general case $\lambda_{k} \in \mathbb{R}_{+}^{*}$ follows by rational approximation.

2nd case: $M$ is hyperfinite. There exists a net $M_{\alpha}$ of finite dimensional subalgebras of the kind as in the 1st case. Further, for every $\alpha$, there exists $J_{\alpha}: M_{\alpha} \rightarrow M$ satisfying the assumptions of lemma 5.5. For every $1<p<\infty$ and every $x \in L^{p}(M), J_{\alpha, p} Q_{\alpha, p} x \stackrel{\alpha}{\rightarrow} x$ in $L^{p}(M)\left(\left[25\right.\right.$, thm 3.4 and rem] and [29, p. 332]). Now (1) of lemma 5.5 yields that $Q_{\alpha} T J_{\alpha}$ is an operator as in the 1st case of the proof. Therefore for any $x \in L^{p}(M)$, by lemma 5.5

$$
\begin{aligned}
\left\langle\left(I_{L^{p}(M)}-T_{p}\right) x, \widehat{x}\right\rangle & =\lim _{\alpha}\left\langle\left(I-T_{p}\right) J_{\alpha, p} Q_{\alpha, p} x,\left(\widehat{J_{\alpha, p} Q_{\alpha, p}} x\right)\right\rangle \\
& =\lim _{\alpha}\left\langle\left(I-Q_{\alpha, p} T_{p} J_{\alpha, p}\right) Q_{\alpha, p} x,\left(\widehat{Q_{\alpha, p} x}\right)\right\rangle \\
& \in \Sigma_{p} .
\end{aligned}
$$


The following theorem now answers our question in section 4 . In addition, the contractivity in (4.4) can be extended to complete contractivity.

Theorem 5.7. Let $M$ be a hyperfinite von Neumann algebra with s.n.f. trace $\tau$ and $\left(T_{t}\right)$ a diffusion semigroup on $(M, \tau)$. Assume that for all $t>0, T_{t}$ satisfies $(\mathcal{P})$ (for example, $T_{t}$ is completely positive). Then for all $1<p<\infty, t \mapsto T_{t, p}$ has an analytic extension

$$
\Sigma_{p}^{\prime} \rightarrow B\left(L^{p}(M)\right), z \mapsto T_{z, p} .
$$

The operators $T_{z, p}$ are in addition completely contractive.

Proof. Proposition 4.2 together with theorem 5.6 gives the analytic extension and the contractivity. To show the complete contractivity, let $n \in \mathbb{N}$ and consider the space $N=M_{n} \otimes M$ with trace $\operatorname{tr} \otimes \tau$. Then $\tilde{T}_{t}:=I_{M_{n}} \otimes T_{t}$ gives a diffusion semigroup on $N$. Further, $\tilde{T}_{t}$ inherits property $(\mathcal{P})$ from $T_{t}$ : Indeed, if $W: M_{2} \otimes M \rightarrow M_{2} \otimes M$ is an "extension" of $T_{t}$ as in the definition of $(\mathcal{P})$, then $I_{M_{n}} \otimes W: M_{n} \otimes\left(M_{2} \otimes M\right) \cong M_{2} \otimes\left(M_{n} \otimes M\right) \rightarrow M_{n} \otimes\left(M_{2} \otimes M\right)$ is one of $\tilde{T}_{t}$. Let $\Sigma_{p}^{\prime} \rightarrow B\left(L^{p}(N)\right), z \mapsto \tilde{T}_{z, p}$ be the analytic contractive extension of $\tilde{T}_{t, p}$. We claim that $\tilde{T}_{z, p}=I_{S_{n}^{p}} \otimes T_{z, p}$, where $T_{z, p}$ is the analytic extension of $T_{t, p}$. Indeed, by the equivalence of the norms $\left\|\left(x_{i j}\right)_{i j}\right\|_{S_{n}^{p}\left(L^{p}(M)\right)} \simeq \sum_{i j}\left\|x_{i j}\right\|_{L^{p}(M)}$, one sees that $I_{S_{n}^{p}} \otimes T_{z}$ is analytic. Since $\tilde{T}_{z, p}=I_{S_{n}^{p}} \otimes T_{z, p}$ a priori for $z>0$, the claim follows from the uniqueness theorem for analytic functions. Now the theorem follows from (3.1).

\section{SPECIFIC EXAMPLES}

We will now give some examples of diffusion semigroups $\left(T_{t}\right)$ on hyperfinite von Neumann algebras which match the conditions of theorem 5.7. Recall that if for any $t>0, T_{t}$ is completely positive, then $T_{t}$ satisfies $(\mathcal{P})$ and theorem 5.7 can be applied. In two specific cases to follow, the complete positivity is unnecessary.

6.1. Commutative case. We assume that $(M, \tau)=\left(L^{\infty}(\Omega), \mu\right)$ is a commutative von Neumann algebra. Then our definition 4.1 of a diffusion semigroup reduces to the classical one given in [28].

For any operator $T: L^{\infty}(\Omega) \rightarrow L^{\infty}(\Omega)$ or $T: L^{1}(\Omega) \rightarrow L^{1}(\Omega),\|T\|=\|T\|_{c b}$. This is false in general for operators $T: L^{p}(\Omega) \rightarrow L^{p}(\Omega)$ with $1<p<\infty$. The property $(\mathcal{P})$ has now a simple characterization.

Proposition 6.1. A $w^{*}$-continuous operator $T: L^{\infty}(\Omega) \rightarrow L^{\infty}(\Omega)$ satisfies $(\mathcal{P})$ if and only if $T$ is contractive and self-adjoint.

Proof. The "only if" part follows from remark 5.2. For the "if" part, we assume that $L^{\infty}(\Omega)=$ $l_{n}^{\infty}$ for some $n \in \mathbb{N}$. The general case can be deduced by an approximation argument as in theorem 5.6, using the semi-partitions of $(\Omega, \mu)$ explained in section 2 .

We identify $T$ and $T_{*}$ with matrices $\left(t_{i j}\right)$ and $\left(\overline{t_{i j}}\right)$. Since $\left(t_{i j}\right)$ is self-adjoint, $\left(\overline{t_{i j}}\right)$ and $\left(\left|t_{i j}\right|\right)$ are self-adjoint also. Hence

$$
W=\left(\begin{array}{cc}
\left(\left|t_{i j}\right|\right) & \left(t_{i j}\right) \\
\left(\overline{t_{j i}}\right) & \left(\left|t_{i j}\right|\right)
\end{array}\right): M_{2}\left(l_{n}^{\infty}\right) \rightarrow M_{2}\left(l_{n}^{\infty}\right)
$$

is self-adjoint. 
We show that $W$ is completely positive. Let $\tilde{J}: l_{n}^{\infty} \hookrightarrow M_{n}$ be the embedding into the diagonal, $J=I_{M_{2}} \otimes \tilde{J}: M_{2}\left(l_{n}^{\infty}\right) \hookrightarrow M_{2}\left(M_{n}\right)$ and $P: M_{2}\left(M_{n}\right) \rightarrow M_{2}\left(l_{n}^{\infty}\right)$ its adjoint. Further, let $\phi_{i j} \in \mathbb{C}$ such that $t_{i j}=\left|t_{i j}\right| \phi_{i j}$. Denote $a_{i j}=\left(\begin{array}{cc}\sqrt{\left|t_{i j}\right|} \phi_{i j} E_{i j} & 0 \\ 0 & \sqrt{\left|t_{i j}\right|} E_{i j}\end{array}\right) \in M_{2}\left(M_{n}\right)$, where $\left(E_{i j}\right)_{i j}$ is the canonical basis in $M_{n}$. Then $x \mapsto \sum_{i, j} a_{i j} x a_{i j}^{*}$ is completely positive by Choi's theorem (3.2). On the other hand, this mapping equals $J W P$. Indeed,

$$
\begin{aligned}
& \sum_{i, j}\left(\begin{array}{cc}
\sqrt{\left|t_{i j}\right|} \phi_{i j} E_{i j} & 0 \\
0 & \sqrt{\left|t_{i j}\right|} E_{i j}
\end{array}\right)\left(\begin{array}{cc}
x^{(11)} & x^{(12)} \\
x^{(21)} & x^{(22)}
\end{array}\right)\left(\begin{array}{cc}
\overline{\phi_{i j}} \sqrt{\left|t_{i j}\right|} E_{j i} & 0 \\
0 & \sqrt{\left|t_{i j}\right|} E_{j i}
\end{array}\right) \\
& =\sum_{i, j}\left(\begin{array}{cc}
\left|t_{i j}\right| E_{i j} x^{(11)} E_{j i} & t_{i j} E_{i j} x^{(12)} E_{j i} \\
\overline{t_{i j}} E_{i j} x^{(21)} E_{j i} & \left|t_{i j}\right| E_{i j} x^{(22)} E_{j i}
\end{array}\right) \\
& =\sum_{i, j}\left(\begin{array}{cc}
\left|t_{i j}\right| x_{j j}^{(11)} E_{i i} & t_{i j} x_{j j}^{(12)} E_{i i} \\
\overline{t_{i j}} x_{j j}^{(21)} E_{i i} & \left|t_{i j}\right| x_{j j}^{(2)} E_{i i}
\end{array}\right) \\
& =J W P x .
\end{aligned}
$$

Then $W=P(J W P) J$ is also completely positive.

As $\|T\|$ is given by $\sup _{i} \sum_{j}\left|t_{i j}\right|$, which does only depend on the absolute values of $t_{i j}$, we have $\left\|\left(\left|t_{i j}\right|\right)\right\|=\|T\|$. This implies $\left\|W\left(\begin{array}{ll}1 & 0 \\ 0 & 1\end{array}\right)\right\| \leq\left\|\left(\left|t_{i j}\right|\right)\right\| \leq 1$, and thus, $W$ is completely contractive.

As a corollary, we obtain [22, cor 3.2], but without the assumption of positivity.

Corollary 6.2. Let $\left(T_{t}\right)$ be a diffusion semigroup on $L^{\infty}(\Omega)$, i.e. the $T_{t, p}$ form consistent contractive $c_{0}$-semigroups on $L^{p}(\Omega)$ for $1 \leq p<\infty\left(w^{*}\right.$-continuous on $\left.L^{\infty}(\Omega)\right)$ such that $T_{t, 2}$ are self-adjoint. Then for $1<p<\infty, t \mapsto T_{t, p}$ has an analytic and contractive extension to

$$
\Sigma_{p}^{\prime}=\left\{z \in \mathbb{C} \backslash\{0\}:|\arg z|<\frac{\pi}{2}-\arctan \frac{|p-2|}{2 \sqrt{p-1}}\right\} .
$$

Proof. Recall that $L^{\infty}(\Omega)$ is a hyperfinite von Neumann algebra. By proposition 6.1, $T_{t}$ satisfies $(\mathcal{P})$ for all $t>0$, so that we can appeal to theorem 5.7.

Remark 6.3. In [19], [22, cor 3.2] is used to improve the angle of the $H^{\infty}$-calculus of generators of commutative diffusion semigroups consisting of positive operators. With the above corollary, [19] gives the same angle improvement without the positivity assumption.

6.2. Schur Multipliers. A further example of non-commutative diffusion semigroups are the Schur multiplier semigroups, considered in [16, chap 8]. The underlying von Neumann algebra is $M=B\left(l^{2}(\mathbb{N})\right)=B\left(l^{2}\right)$, with the usual trace $\operatorname{tr}$. We identify $B\left(l^{2}(\mathbb{N})\right)$ with some subspace of $\mathbb{C}^{\mathbb{N} \times \mathbb{N}}$ in the usual way. Let $\left(t_{i j}\right)_{i j} \in \mathbb{C}^{\mathbb{N} \times \mathbb{N}}$. The Schur multiplier $T$ associated with $\left(t_{i j}\right)_{i j}$ is defined in the following way: If $x=\left(x_{i j}\right)_{i j} \in B\left(l^{2}\right)$ then

$$
T x=\left(t_{i j} x_{i j}\right)_{i j} \text {. }
$$

Of course, it is not sure that $T x \in B\left(l^{2}\right)$ nor that $T \in B(M)$. The following proposition characterizes, when the latter is the case. For a proof, see [24, cor 8.8]. 
Proposition 6.4. Let $T$ be given by (6.1). The following are equivalent.

- There exists a Hilbert space $H$ and sequences $\left(x_{i}\right)_{i},\left(y_{i}\right)_{i} \subset H$ such that $\sup _{i}\left\|x_{i}\right\| \leq$ $1, \sup _{i}\left\|y_{i}\right\| \leq 1$ and $t_{i j}=\left\langle x_{i}, y_{j}\right\rangle_{H}$.

- The Schur multiplier $T$ is a bounded operator on $M$ and $\|T\| \leq 1$.

- The Schur multiplier $T$ is a completely bounded operator on $M$ and $\|T\|_{c b} \leq 1$.

Assume now that the conditions of the above proposition are satisfied. Then for $x, y \in$ $S^{1} \cap B\left(l^{2}\right),\left\langle T x, y^{*}\right\rangle=\operatorname{tr}\left(T x y^{*}\right)=\sum_{i, j=1}^{\infty} t_{i j} x_{i j} \overline{y_{i j}}$. Therefore, $T$ is self-adjoint if and only if $t_{i j} \in \mathbb{R}$ for all $i, j \in \mathbb{N}$.

Lemma 6.5. A Schur multiplier $T: B\left(l^{2}\right) \rightarrow B\left(l^{2}\right)$ satisfies $(\mathcal{P})$ if and only if $T$ is contractive and self-adjoint.

Proof. Only the "if" part has to be shown. Let $\left(x_{i}\right)_{i},\left(y_{i}\right)_{i} \subset H$ be the sequences given as in proposition 6.4. By the self-adjointness of $T$, we know that $\left\langle x_{i}, y_{j}\right\rangle_{H} \in \mathbb{R}$. We may suppose that $\left\langle x_{i}, x_{j}\right\rangle_{H},\left\langle y_{i}, y_{j}\right\rangle_{H} \in \mathbb{R}$.

Indeed, if this is not the case, let $\left(e_{\gamma}\right)_{\gamma}$ be an orthonormal basis of $H$ and consider the $\mathbb{R}$-linear mapping

$$
J:\left\{\begin{array}{ll}
H & \longrightarrow H \oplus_{2} H \\
e_{\gamma} & \longmapsto e_{\gamma} \oplus 0 \\
i e_{\gamma} & \longmapsto 0 \oplus e_{\gamma}
\end{array} .\right.
$$

For $x \in H$, write $x=x_{R}+i x_{I}$, where $x_{R}$ and $x_{I}$ are in the real span of the $e_{\gamma}^{\prime} s$. In the same manner, write $y=y_{R}+i y_{I}$. Then

$$
\langle x, y\rangle_{H}=\left\langle x_{R}, y_{R}\right\rangle_{H}+\left\langle x_{I}, y_{I}\right\rangle_{H}+i\left\langle x_{I}, y_{R}\right\rangle_{H}-i\left\langle x_{R}, y_{I}\right\rangle_{H}
$$

so if $\langle x, y\rangle_{H} \in \mathbb{R}$, then $\langle J(x), J(y)\rangle_{H \oplus H}=\langle x, y\rangle_{H}$. Replace now $x_{i}$ and $y_{i}$ by $J\left(x_{i}\right)$ and $J\left(y_{i}\right)$. Then, we still have $t_{i j}=\left\langle J\left(x_{i}\right), J\left(y_{j}\right)\right\rangle$, and in addition $\left\langle x_{i}, x_{j}\right\rangle_{H},\left\langle y_{i}, y_{j}\right\rangle_{H} \in \mathbb{R}$.

The operator $W$ as in definition 5.1 that we will give in a moment acts on the space $M_{2} \otimes B\left(l^{2}\right)$. We wish to consider Schur multipliers on this space and do this in virtue of the natural identification $M_{2} \otimes B\left(l^{2}\right) \cong B\left(l^{2}(\mathbb{N} \times\{1,2\})\right)$. Note that $T_{*}$ is the Schur multiplier associated with $\left(\left\langle y_{i}, x_{j}\right\rangle_{H}\right)_{i j}$. Further, by proposition 6.4 , the Schur multipliers $S_{1}$ and $S_{2}$ associated with $\left(\left\langle x_{i}, x_{j}\right\rangle_{H}\right)_{i j}$ and $\left(\left\langle y_{i}, y_{j}\right\rangle\right)_{i j}$ are completely contractive. We put $W=\left(\begin{array}{ll}S_{1} & T \\ T_{*} & S_{2}\end{array}\right)$. This is a Schur multiplier on $M_{2} \otimes B\left(l^{2}\right) \cong B\left(l^{2}(\mathbb{N} \times\{1,2\})\right)$ associated with the matrix

$$
\left(\left\langle z_{(i k)}, z_{(j l)}\right\rangle_{H}\right)_{(i k),(j l) \in \mathbb{N} \times\{1,2\}},
$$

where $z_{(i k)}=\left\{\begin{array}{ll}x_{i}, & k=1 \\ y_{i}, & k=2\end{array}\right.$. Therefore, $W$ is completely positive [24, ex 8.7]. The (complete) contractivity of $W$ is clear from proposition 6.4. Finally, as $\left\langle z_{(i k)}, z_{(j l)}\right\rangle_{H} \in \mathbb{R}, W$ is selfadjoint.

Now assume that $\left(T_{t}\right)$ is a diffusion semigroup on $M$ such that for any $t>0, T_{t}$ is a Schur multiplier associated to some $\left(t_{i j}^{(t)}\right)_{i j} \in \mathbb{C}^{\mathbb{N} \times \mathbb{N}}$. For example, if $H$ is a Hilbert space and $\left(\alpha_{k}\right)_{k \in \mathbb{N}}$ and $\left(\beta_{k}\right)_{k \in \mathbb{N}}$ are sequences in $H$, then the Schur multipliers $T_{t}$ associated with 
$\left(e^{-t\left\|\alpha_{i}-\beta_{j}\right\|}\right)_{i j}$ form such a diffusion semigroup [16, prop 8.17]. Then the above lemma and theorem 5.7 show that for any $1<p<\infty,\left(T_{t, p}\right)_{t>0}$ admits an analytic extension

$$
\Sigma_{p}^{\prime} \rightarrow B\left(L^{p}\right), z \mapsto T_{z, p}
$$

Further, by the uniqueness of analytic vector valued functions, $T_{z, p}$ is again a Schur multiplier for any $z \in \Sigma_{p}^{\prime}$.

\section{Semi-COmmutative Diffusion Semigroups}

At the end, we give an example of a diffusion semigroup on a von Neumann algebra without the assumption of hyperfiniteness. Let $(\Omega, \mu)$ be a measure space and $(N, \sigma)$ a von Neumann algebra with s.n.f. trace. Suppose we are given a diffusion semigroup $\left(T_{t}\right)$ on $L^{\infty}(\Omega)$. By the $w^{*}$-continuity of any $T_{t}$, we can define the contractions

$$
T_{t}^{N}:=T_{t} \bar{\otimes} I_{N}: L^{\infty}(\Omega) \bar{\otimes} N \rightarrow L^{\infty}(\Omega) \bar{\otimes} N .
$$

$\left(T_{t}^{N}\right)$ is a diffusion semigroup on $L^{\infty}(\Omega) \bar{\otimes} N$, and called semi-commutative diffusion semigroup.

Now assume that $N$ has the QWEP property. This means that $N$ is the quotient of a $C^{*}$ algebra having the weak expectation property (WEP) introduced in [20, 21]. It is unknown whether every von Neumann algebra has this property.

Recall the following notion of an ultraproduct of Banach spaces. Let $\left(X_{\alpha}\right)_{\alpha \in I}$ be a family of Banach spaces and $\mathcal{U}$ an ultrafilter on $I$. We will only need the case $X_{\alpha}=X$, a fixed Banach space. Consider the quotient space

$$
l^{\infty}\left(I ; X_{\alpha}\right)=\left\{\left(x_{\alpha}\right)_{\alpha} \in \prod_{\alpha} X_{\alpha}: \sup _{\alpha}\left\|x_{\alpha}\right\|<\infty\right\} /\left\{\left(x_{\alpha}\right)_{\alpha}: \exists F \in \mathcal{U}: x_{\alpha}=0 \text { for all } \alpha \in F\right\}
$$

and the subspace

$$
c_{0}\left(\mathcal{U} ; X_{\alpha}\right)=\left\{\left(x_{\alpha}\right)_{\alpha} \in l^{\infty}\left(I ; X_{\alpha}\right): \lim _{\mathcal{U}}\left\|x_{\alpha}\right\|=0\right\} .
$$

Then $\prod_{\mathcal{U}} X_{\alpha}=l^{\infty}\left(I ; X_{\alpha}\right) / c_{0}\left(\mathcal{U} ; X_{\alpha}\right)$ is called an ultraproduct, see also [26, p. 59].

We will need a property of $L^{p}(N, \sigma)$ which appears in [13].

Proposition 7.1. Let $N$ be a von Neumann algebra with QWEP having a s.n.f. trace $\sigma$. Then there exists a Hilbert space $H$, an ultrafilter $\mathcal{U}$ on some index set $I$ and an isometric embedding $J: L^{p}(N) \rightarrow \prod_{\mathcal{U}} S^{p}(H)$.

The following proposition follows from [13, thm 2.10]. We include a simple proof for the convenience of the reader.

Proposition 7.2. Let $1<p<\infty, L^{p}(\Omega)$ be some commutative $L^{p}$-space and $T \in B\left(L^{p}(\Omega)\right)$ be completely bounded. Let $N$ be a von Neumann algebra with QWEP with a s.n.f. trace $\sigma$. Then $T \otimes I_{L^{p}(N)}$, initially defined on $L^{p}(\Omega) \otimes L^{p}(N)$, extends to $L^{p}\left(\Omega, L^{p}(N)\right)$ and

$$
\left\|T \bar{\otimes} I_{L^{p}(N)}: L^{p}\left(\Omega, L^{p}(N)\right) \rightarrow L^{p}\left(\Omega, L^{p}(N)\right)\right\| \leq\|T\|_{c b} .
$$


Proof. By (3.1), for every $n \in \mathbb{N}$,

$$
\left\|T \otimes I_{S_{n}^{p}}: L^{p}\left(\Omega, S_{n}^{p}\right) \rightarrow L^{p}\left(\Omega, S_{n}^{p}\right)\right\| \leq\|T\|_{c b}
$$

As in [25, prop 2.4], we deduce via a density argument that $\| T \bar{\otimes} I_{S^{p}(H)}: L^{p}\left(\Omega, S^{p}(H)\right) \rightarrow$ $L^{p}\left(\Omega, S^{p}(H)\right)\|\leq\| T \|_{c b}$.

Let $H, \mathcal{U}, I, J$ be as in proposition 7.1. We denote $\left(x_{\alpha}\right)_{\alpha}$ and $\left(f_{\alpha}\right)_{\alpha}$ elements of the ultraproduct spaces $\prod_{\mathcal{U}} S^{p}(H)$ and $\prod_{\mathcal{U}} L^{p}\left(\Omega, S^{p}(H)\right)$. Consider the ultraproduct mapping

$$
S: \prod_{\mathcal{U}} L^{p}\left(\Omega, S^{p}(H)\right) \rightarrow \prod_{\mathcal{U}} L^{p}\left(\Omega, S^{p}(H)\right),\left(f_{\alpha}\right)_{\alpha} \mapsto\left(\left(T \bar{\nabla} I_{S^{p}(H)}\right)\left(f_{\alpha}\right)\right)_{\alpha} .
$$

Note that the space $L^{p}\left(\Omega, \prod_{\mathcal{U}} S^{p}(H)\right)$ is isometrically embedded in $\prod_{\mathcal{U}} L^{p}\left(\Omega, S^{p}(H)\right)$, via a mapping taking a step function $\sum_{k} f_{k} \otimes\left(x_{k, \alpha}\right)_{\alpha}$ to the element $\left(\sum_{k} f_{k} \otimes x_{k, \alpha}\right)_{\alpha}$. With this embedding, $S\left(L^{p}\left(\Omega, \prod_{\mathcal{U}} S^{p}(H)\right)\right) \subset L^{p}\left(\Omega, \prod_{\mathcal{U}} S^{p}(H)\right)$, and $\tilde{S}=\left.S\right|_{L^{p}\left(\Omega, \prod_{\mathcal{U}} S^{p}(H)\right)}$ is again a contraction, since $\|T\|_{c b} \leq 1$. Now use proposition 7.1 to restrict $\tilde{S}$ to $L^{p}\left(\Omega, L^{p}(N)\right)$ ). This restriction equals $T \bar{\otimes} I_{L^{p}(N)}$, which is thus a contraction, as desired.

Corollary 7.3. Let $\left(T_{t}^{N}\right)=\left(T_{t} \bar{\otimes} I_{N}\right)$ be a semi-commutative diffusion semigroup as above. Then for $1<p<\infty, t \mapsto T_{t, p}^{N}$ has an analytic and completely contractive extension to $\Sigma_{p}^{\prime}$.

Proof. By proposition 6.1, $T_{t}$ satisfies $(\mathcal{P})$ and theorem 5.7 gives the completely contractive analytic extension $z \mapsto T_{z, p}$ on $\Sigma_{p}^{\prime}$. Now appeal to proposition 7.2 to get the contractive operators $T_{z, p} \bar{\otimes} I_{L^{p}(N)}$. It is clear that the latter form an analytic extension of $T_{t, p}^{N}$. Replacing $T_{t}$ by $I_{M_{n}} \otimes T_{t}$ in this argument gives the completely contractive result.

Remark 7.4. There is even a more general version of proposition 7.2, [13, thm 2.10]. From this, we deduce that if $M$ is a hyperfinite von Neumann algebra with s.n.f. trace $\tau$ and $T: L^{p}(M) \rightarrow L^{p}(M)$ is completely contractive, then $T \bar{\otimes} I_{L^{p}(N)}: L^{p}(M \bar{\otimes} N) \rightarrow L^{p}(M \bar{\otimes} N)$ is completely contractive.

With this generalization, one also gets the following result: If $\left(T_{t}\right)$ is a diffusion semigroup on a hyperfinite von Neumann algebra such that $T_{t}$ satisfies $(\mathcal{P})$ for all $t>0$, then $T_{t}^{N}=$ $T_{t} \bar{\otimes} I_{N}$ forms a diffusion semigroup and has an analytic and completely contractive extension to $\Sigma_{p}^{\prime}$.

Corollary 7.3 allows us to generalize proposition 4.4, which was our starting observation, to the non-commutative case.

Corollary 7.5. Let $(N, \sigma)$ be a QWEP von Neumann algebra and $a, b \in L^{p}(N)$. Then

$$
\langle a-b, \widehat{a}-\widehat{b}\rangle=\|a\|_{p}^{p}+\|b\|_{p}^{p}-\operatorname{tr}\left(b|a|^{p-1} u_{a}\right)-\operatorname{tr}\left(a|b|^{p-1} u_{b}\right) \in \Sigma_{p} .
$$

Here, $a=u_{a}|a|$ and $b=u_{b}|b|$ are the polar decompositions.

Proof. Let $\left(T_{t}\right)$ be the diffusion semigroup on $l_{2}^{\infty}$ as in example 4.3, i.e. $T_{t}=e^{t A}$ with

$$
A=\left(\begin{array}{cc}
-1 & 1 \\
1 & -1
\end{array}\right)
$$

Consider the semi-commutative semigroup $\left(T_{t} \bar{\otimes} I_{N}\right)$ with (bounded) generator $A_{p}=A \otimes$ $I_{L^{p}(N)}$ on $L^{p}\left(l_{2}^{\infty} \otimes N\right)$ and define the element $x$ in this space by $x=(a, b)$. Its dual element 
is given by $\widehat{x}=(\widehat{a}, \widehat{b})$. By corollary 7.3 and proposition 4.2 ,

$$
\langle a-b, \widehat{a}-\widehat{b}\rangle=-\left\langle A_{p} x, \widehat{x}\right\rangle \in \Sigma_{p} .
$$

\section{ACKNOWLEDGEMENTS}

The author would like to thank Christian Le Merdy for stimulating discussions on this subject and for improving the presentation of the article, and Éric Ricard for showing him the example 5.3.

\section{REFERENCES}

[1] D. Bakry, Sur l'interpolation complexe des semigroupes de diffusion, Séminaire de probabilités XXIII, Lect. Notes Math. 1372, 1-20 (1989).

[2] J. Bergh, J. Löfström, Interpolation spaces. An introduction, Grundlehren der math. Wiss. 223. BerlinHeidelberg-New York: Springer-Verlag. X, 207 p. with 5 figs (1976).

[3] D. Blecher, C. Le Merdy, Operator algebras and their modules - an operator space approach. LMS, Monographs, New Series, 30., Oxford University Press, x+387 p. (2004).

[4] M. Choi, Completely positive linear maps on complex matrices, Linear Algebra Appl. 10, 285-290 (1975).

[5] R. Chill, E. Fasangova, G. Metafune, D. Pallara, The sector of analyticity of the Ornstein-Uhlenbeck semigroup on $L^{p}$ spaces with respect to invariant measure, J. Lond. Math. Soc., II. Ser. 71, No.3, 703-722 (2005).

[6] R. Chill, E. Fasangova, G. Metafune, D. Pallara, The sector of analyticity of nonsymmetric submarkovian semigroups generated by elliptic operators, C. R., Math., Acad. Sci. Paris 342, No.12, 909-914 (2006).

[7] M. Cowling, Harmonic analysis on semigroups, Ann. Math. (2) 117, 267-283 (1983).

[8] E. Davies, One parameter semigroups, LMS, Monographs, No.15. London: Academic Press, Publishers. VIII, 230 p. (1980).

[9] E. Effros, Z. Ruan, Operator spaces, LMS, Monographs, No.23. Oxford: Clarendon Press. xvi+363 p. (2000).

[10] J. Goldstein, Semigroups of Linear Operators and Applications. Oxford Math. Mon. New York: Oxford University Press, X, 245 p. (1985).

[11] T. Hytönen, Littlewood-Paley-Stein theory for semigroups in UMD spaces, Rev. Mat. Iberoam. 23, No.3, 973-1009 (2007).

[12] U. Haagerup, Injectivity and decomposition of completely bounded maps, Operator algebras and their connections with topology and ergodic theory, Proc. Conf., Buşteni/Rom. 1983, Lect. Notes Math. 1132, 170-22 (1985)

[13] M. Junge, Applications of the Fubini theorem for non-commutative $L^{p}$ spaces, preprint.

[14] M. Junge, Q. Xu, Théorèmes ergodiques maximaux dans les espaces $L_{p}$ non commutatifs, C.R. Math. Acad. Sci. Paris 334, No.9, 773-778 (2002).

[15] M. Junge, Q. Xu, Noncommutative maximal ergodic theorems, J. Am. Math. Soc. 20, No.2, 385-439 (2007).

[16] M. Junge, C. Le Merdy, Q. Xu, $H^{\infty}$ functional calculus and square functions on noncommutative $L^{p}$ spaces, Astérisque 305. Paris: Société Mathématique de France. vi+138 p. (2006).

[17] P. Kunstmann, Uniformly elliptic operators with maximal $L^{p}$-spectrum in planar domains, Arch. Math. 76, No.5, 377-384 (2001).

[18] P. Kunstmann, $L^{p}$-spectral properties of the Neumann Laplacian on horns, comets and stars, Math. Z. 242, No.1, 183-201 (2002).

[19] P. Kunstmann, Ž. Štrkalj, $H^{\infty}$-calculus for submarkovian generators, Proc. Am. Math. Soc. 131, No.7, 2081-2088 (2003).

[20] C. Lance, On nuclear $C^{*}$-algebras, J. Funct. Anal. 12, 157-176 (1973). 
[21] C. Lance, Tensor products and nuclear $C^{*}$-algebras, Operator algebras and applications, Proc. Symp. Pure Math. 38, Part 1, Kingston/Ont. 1980, 379-399 (1982).

[22] V. Liskevich, M. Perelmuter, Analyticity of submarkovian semigroups, Proc. Am. Math. Soc. 123, No.4, 1097-1104 (1995).

[23] N. Okazawa, Sectorialness of Second order elliptic operators in divergence form, Proc. Am. Math. Soc. 113, No.3, 701-706 (1991).

[24] V. Paulsen, Completely Bounded Maps and Operator Algebras, Cambridge Studies in Advanced Mathematics. 78. Cambridge: Cambridge University Press. xii+300 p. (2002).

[25] G. Pisier, Non-commutative vector valued $L^{p}$ spaces and completely p-summing maps, Astérisque. 247. Paris: Société Mathématique de France, iv+130 p. (1998).

[26] G. Pisier, Introduction to Operator space theory, LMS Lecture Note Series 294. Cambridge: Cambridge University Press. vii+478 p. (2003).

[27] G. Pisier, Q. Xu, Non-commutative $L^{p}$ spaces, Handbook of the geometry of Banach spaces. Volume 2. Amsterdam: North-Holland. 1459-1517 (2003).

[28] E. Stein, Topics in Harmonic Analysis related to the Littlewood-Paley Theory, Annals of Mathematics Studies. 63. Princeton, N. J.: Princeton U. P. and the U. of Tokyo Press, VIII, 146 p. (1970).

[29] M. Takesaki, Theory of operator algebras I, Encycl. of Math. Sc. Operator Algebras and NonCommutative Geometry. 124(5). Berlin: Springer. xix +415 p. (2002).

[30] M. Terp, $L^{p}$-spaces associated with von Neumann algebras, Notes, Math. Institute, Copenhagen university (1981).

[31] J. Voigt, The sector of holomorphy for symmetric submarkovian semigroups, Functional analysis. Proceedings of the first international workshop held at Trier University, Germany, Berlin: de Gruyter. 449-453 (1996).

[32] J. Voigt, Abstract Stein interpolation, Math. Nachr. 157, 197-199 (1992).

Institut Für Analysis, Kaiserstrasse 89, 76133 Karlsruhe, Germany

Laboratoire de Mathématiques, Université de Franche-Comté, 25030 Besançon Cedex, FRANCE

E-mail address: christoph.kriegler@univ-fcomte.fr 